\title{
Evaluating the effects of smoking and smoking cessation on alveolar bone in young adults: An interventional prospective study
}

\author{
Hiral A. Parikh ${ }^{*}$, Mihir N. Shah², Sujay B. Shah ${ }^{3}$, Pratik S. Shah ${ }^{4}$, Rahul R. Patel ${ }^{5}$ \\ ${ }^{1,3} \mathrm{Ph} . \mathrm{D}$ Scholar, ${ }^{2}$ Dean, Professor \& Head, ${ }^{4}$ Private Practitioner, ${ }^{5}$ Reader, ${ }^{1,3}$ Gujarat University, Ahmedabad, Gujarat, ${ }^{2}$ Dental College \& \\ Hospital, Gandhinagar, Gujarat, ${ }^{4}$ Private Practitioner, Surendranagar, Gujarat, ${ }^{5}$ Narsinhbhai Patel Dental College \& Hospital, Visnagar, \\ Gujarat, India
}

\section{*Corresponding Author: Hiral A. Parikh}

Email: drhiral10@yahoo.co.in

\begin{abstract}
Introduction: Preponderance of evidence suggests that smoking is one of the most significant risk factors in the development and progression of periodontal diseases. Smoke contains thousands of different compounds like Nicotine, Tar. Lack of education of dentists and hygienists to promote smoking quitting activities is a significant barrier for Motivational Interviewing for smokers.

Aim: To evaluate the effect of smoking on alveolar bone in young adults and motivating patients to quit through the Motivational Interviewing.

Materials and Methods: Sixty subjects were categorized into three groups. Group 1: smokers, Group 2:non-smokers, Group 3: smokers but willing to quit smoking.For alveolar bone height measurement Image Digitization was done.Smoking cessation was carried out for Group 3. Motivational Interviewing was used to convince the patients for smoking cessations. After six months, Nicoscreen ${ }^{\circledR}$ was used to know whether they quitted smoking or not.

Results: Group 1 had statistically significant bone loss in comparison to Group 2 \& Group 3 after six months which is attributed to effect of smoking on alveolar bone.

Conclusion: Periodontal health is compromised by chronic smoking by an increase alveolar bone loss. Nicoscreen ${ }^{\circledR}$ can be used as a prompt screening tool for smoking cessation.
\end{abstract}

Keywords: Smoking, Motivational interviewing, Smoking cessation.

\section{Introduction}

Periodontitis is the most prevalent form of periodontal diseases which is multifactorial in nature but bacterial infection being the main culprit. In addition, there are several risk factors i.e. smoking, diabetes (systemic diseases), stress etc. that may increase the probability and severity of periodontitis. ${ }^{1}$

Preponderance of evidence suggests that smoking is one of the most significant risk factors in the development and progression of periodontal diseases. ${ }^{2}$ The postulated mechanisms of increased periodontal disease progression in smokers include alterations in the host response, such as reductions in serum $\mathrm{IgG}_{2}$ levels and impairment of various neutrophils functions, such as phagocytosis and aerobic antimicrobial functions. ${ }^{3}$ Other reported effects of smoking on the periodontium are suppression of fibroblast and osteoblasts functions, alterations in gingival blood flow, and reductions in the GCF flow. ${ }^{4}$ It has been well documented that smoking adversely affects boneby reducing bone mineral content. ${ }^{5}$

The association between cigarette smoking and periodontal diseases represents a significant oral health problem. Smoke contains thousands of different compounds like Nicotine, "Tar" (composed of many chemicals), Benzene, Benzo pyrene. Cotinine, a metabolite of nicotine, can be measured in the serum/plasma, urine and saliva, and is a better measure of cigarette smoke exposure as it has a longer half-life than nicotine (18 hrs compared with 1-2 hrs). Smokers would be expected to have serum cotinine levels of over $14 \mathrm{ng} / \mathrm{ml}$, and this could be as high as 1000 $\mathrm{ng} / \mathrm{ml}$. Resting plasma nicotine levels are much lower (5-50 $\mathrm{ng} / \mathrm{ml}$ ), and are maintained by the individual to satisfy their craving for nicotine.

Lack of education of dentists and hygienists in terms of being able to select effective treatments available to promote quitting activities is a significant barrier for Motivational Interviewing (MI) for smokers. Various oral symptoms and dental treatments relevant to smoking may be applicable to motivate patients in dental clinics. The theoretical model for intervention with respect to behavioural approaches involves stage-based interventions. ${ }^{6}$ This model separates smokers into five different stages: precontemplation, contemplation, preparation, action, and maintenance. Progression through these stages is sequential, although relapse to an earlier stage could occur.

Several types of tobacco dependence treatments involving counselling, behavioural therapy, and pharmacotherapy should be employed in all smokers who attempt quitting.

The aim of this study was to evaluate the effect of smoking on alveolar bone in young adults with a few years of smoking and motivating patients to quit and promoting for smoking cessation through the Motivational Interviewing (MI). The objectives of the study were to compare effect of smoking with clinical parameters, to compare crestal bone loss in smokers, non-smokers and smoking cessation groups, to evaluate the role of smoking cessation by Motivational Interviewing (MI) and to evaluate Nicoscreen ${ }^{\circledR}$ as a screening tool for smoking cessation. 


\section{Methodology}

It was an interventional, prospective and comparative study, carried out on patients selected from the outpatient Department of Periodontics \& Oral Implantology, Ahmedabad Dental College and Hospital for evaluation of the effect of smoking and smoking cessation on alveolar bone.

Patients were randomly selected based on following criteria. The subjects included only males aged 18-26 years. Subjects having all premolars, molars and lower central incisors without mal alignment/ malocclusion were included. Subjects considered not having periodontitis as identified by clinical examination by following criteria:1) No more than one tooth with inter proximal attachment loss $\geq 2 \mathrm{~mm}$. 2) Site should not have probing depth more than $3 \mathrm{~mm}$. 3) Subjects should not have attachment loss associated with recession.

The subjects having any systemic condition that affect periodontal condition i.e. diabetes, individual who have received periodontal therapy before 12 months from baseline of study, antibiotics or NSAID within 6 months prior to study, subjects who have undergone orthodontic treatment and patient with diastema were excluded from study.

The subjects once selected, underwent routine periodontal examination. Cigarette smoking status was obtained from a written questionnaire completed by each subject, which was reported to be a valid method for estimating smoking prevalence. Subjects were considered smokers if they had been smoking $>5$ cigarettes per day since last 2 years. ${ }^{7}$

A total number of sixty patients participated in this study. They were categorized into three Groups. Group 1 $(n=20)$ consisteds of smokers, Group $2(n=20)$ consisteds of non-smokers and Group 3 ( $n=16)$ consisteds of patients who were smokers but willing to quit smoking. Former smokers were not included in the study.

A parallel-arm prospective design for the study was used. After enrollment in the study, all individuals were evaluated clinically and radiographically (baseline evaluation). Then, with the purpose of obtaining a uniform periodontal status, all subjects underwent a rigorous dental hygiene programme (DHP). The DHP consisted of approximately two sessions per week, for 3 weeks, of oral hygiene instructions (brushing and flossing), scaling (quadrant by quadrant at 1-week intervals and using selected hand instruments), and polishing with rotary instruments. The endpoint of the mechanical treatment included removal of supragingival and subgingival calculus and stain to achieve a smooth surface. Fifteen days later, a second clinical evaluation was performed to assess the effect of the DHP on baseline periodontal status. After this, each subject continued throughout the study with a personal oral hygiene routine (as instructed during the DHP) and with no professional periodontal maintenance. At the end of the study, periodontal therapy was performed as needed. A reported change in smoking habits (reviewed at each session). After six months clinical and radiographic parameters were re-measured.

For the radiographic measurements, RVG were taken by using standardised parallel device. Only one dental x-ray equipment Gomax ${ }^{\circledR}$ was used, with the exposure parameters fixed at $60 \mathrm{kV}(\mathrm{p}), 15 \mathrm{~mA}$, and 1 second. For each subject, three RVG were taken: left \& right from the molarpremolar region and one at mandibular anterior region between two central incisors.

Image Digitization was done by transferring the RVGto Sopro $^{\circledR}$ software for analysis and measurement. After transferring the image, brightness was adjusted to identify CEJ. Digitized images were analyzed with a software program using a magnification in a 15 -inch non-interlaced high-resolution monitor with a display resolution of $800 \times$ 600 pixels. All image evaluations were performed by a single operator, seated $50 \mathrm{~cm}$ far from the screen in a room with soft light without prior knowledge of the experimental group to which each image belonged.

For bone height measurement, the distance from the cemento-enamel junction (CEJ) to the alveolar bone crest $(\mathrm{ABC})$ was measured in millimetres at three proximal sites per image: one molar-molar site referred to distal surfaces of first molars, one molar-premolar site referred to mesial surfaces of first molars, and one premolar-premolar site referred to mesial surfaces of second premolars. Because two (left/right) radiographs were taken from the molarpremolar region, a total of six posterior proximal sites (Fig. 1) and one in lower anterior between two central incisors (Fig. 2) per subject were measured at each evaluation point over the six months of period. The observer performing the analysis identified and marked the CEJ and then identified and marked the $\mathrm{ABC}$ as the most coronal position where the periodontal ligament space was uniform in thickness.

Smoking cessation was carried out after baseline clinical and radiographic measurement for Group 3. Motivational Interviewing (MI) was used to convince the patients for smoking cessations. After six months special cessation kit Nicoscreen ${ }^{\circledR}$ (Fig. 3) was used to know actual status of patients whether they had quit smoking or not.

Nicoscreen ${ }^{\circledR}$ is based on the principle of the highly specific immunochemical reactions between antigens and antibodies, which are used for the analysis of specific substances in urine. The drops of urine are placed onto a cassette and the assay reacts within 5 minutes providing preliminary evidence of nicotine use by appearance of line that indicate positive or negative result.

Interpretation of results (Fig. 4,5): Negative: Two Colour Bands: The appearance of two purple bands within the result window indicates a negative test result. Positive: One Colour Band: The appearance of only the control line, ' $\mathrm{C}$ ', within the result window indicates the result is positive, i.e. the specimen contains COT at a concentration above the cut-off level. Invalid: No Band- A distinct control line, ' $\mathrm{C}$ ', should always appear in the result window. The test is invalid if no control line forms in the result window. 


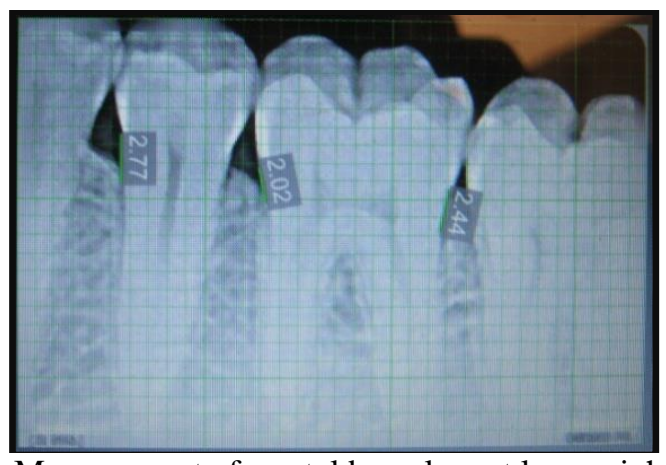

Fig. 1: Measurement of crestal bone loss at lower right posterior region

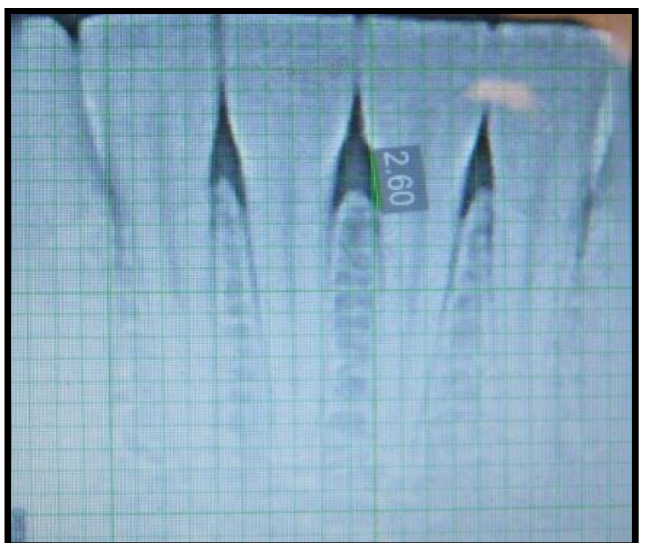

Fig. 2: Measurement of crestal bone loss at mesial surface of mandibular right central incisor

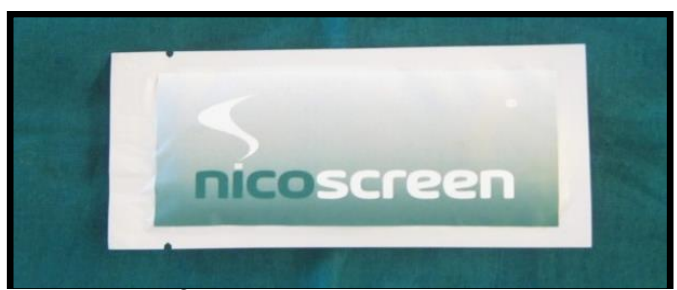

Fig. 3: Nicoscreen ${ }^{\circledR}$ (Kit for smoking cessation)

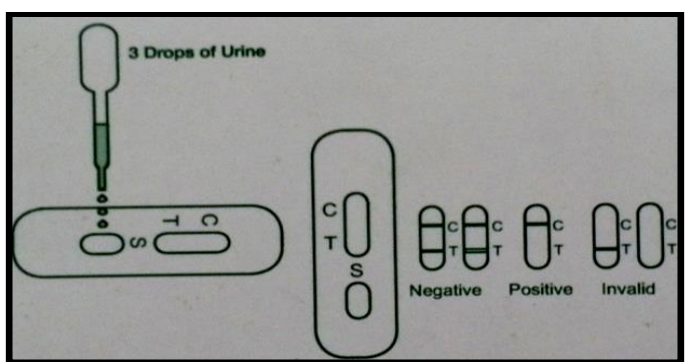

Fig. 4: Nicoscreen ${ }^{\circledR}$ reaction

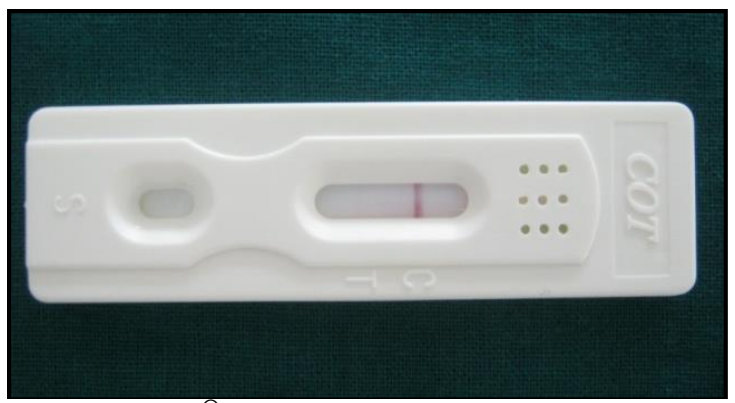

Fig. 5: Nicoscreen ${ }^{\circledR}$ shows positive result

\section{Results}

All the data were transferred to SPSS v17 for the statistical analysis. The Student's $t$-test for paired comparisons was used to determine the significance of the difference between initial and re-evaluation clinical parameters and measurement of bone loss for the study subjects. Analysis of variance (ANOVA) was used to compare multiple groups for a measurement of bone loss and clinical parameter (i.e. PI and GI) among all the groups. Multiple comparisons were done between the group forms using the Tukey's test for measurement of bone loss and clinical parameter (i.e. PI and GI). (Table 1-3)

Table 1: Inter-group comparison of site specific mean crestal bone loss

\begin{tabular}{|c|c|c|c|c|c|c|}
\hline \multirow[t]{2}{*}{ Site } & & \multicolumn{4}{|c|}{ Groups } & \multirow[b]{2}{*}{$p$ value } \\
\hline & & Group 1 & Group 2 & Group 3 & F value & \\
\hline \multirow{2}{*}{$\begin{array}{l}\text { Right side distal surface of } \\
1^{\text {st }} \text { molar }\end{array}$} & Baseline & $1.98 \pm 0.55$ & $1.55 \pm 0.40$ & $2.36 \pm 0.42$ & 12.64 & $<0.001 * *$ \\
\hline & At 6 months & $2.26 \pm 0.60$ & $1.57 \pm 0.41$ & $2.38 \pm 0.41$ & 14.38 & $<0.001^{* * *}$ \\
\hline \multirow{2}{*}{$\begin{array}{l}\text { Right side mesial surface of } \\
\qquad 1^{\text {st }} \text { molar }\end{array}$} & Baseline & $2.00 \pm 0.81$ & $1.59 \pm 0.42$ & $2.28 \pm 0.41$ & 5.85 & 0.005 \\
\hline & At 6 months & $2.33 \pm 0.83$ & $1.62 \pm 0.43$ & $2.30 \pm 0.42$ & 8.06 & 0.001 \\
\hline \multirow{2}{*}{$\begin{array}{c}\text { Right side mesial surface of } \\
2^{\text {nd }} \text { pre-molar }\end{array}$} & Baseline & $2.10 \pm 0.65$ & $1.73 \pm 0.45$ & $2.29 \pm 0.42$ & 5.06 & 0.01 \\
\hline & At 6 months & $2.48 \pm 0.59$ & $1.71 \pm 0.47$ & $2.31 \pm 0.41$ & 12.39 & $<0.001 * *$ \\
\hline \multirow{2}{*}{$\begin{array}{l}\text { Left side distal surface of } \\
1^{\text {st }} \text { molar }\end{array}$} & Baseline & $2.04 \pm 0.56$ & $1.66 \pm 0.30$ & $2.27 \pm 0.34$ & 9.04 & $<0.001^{* *}$ \\
\hline & At 6 months & $2.28 \pm 0.50$ & $1.68 \pm 0.30$ & $2.29 \pm 0.34$ & 14.4 & $<0.001^{* * *}$ \\
\hline \multirow{2}{*}{$\begin{array}{c}\text { Left side mesial surface of } \\
1^{\text {st }} \text { molar }\end{array}$} & Baseline & $1.92 \pm 0.63$ & $1.77 \pm 0.33$ & $2.34 \pm 0.34$ & 6.09 & 0.004 \\
\hline & At 6 months & $2.30 \pm 0.70$ & $1.79 \pm 0.33$ & $2.35 \pm 0.35$ & 6.77 & 0.002 \\
\hline \multirow{2}{*}{$\begin{array}{l}\text { Left side mesial surface of } \\
2^{\text {nd }} \text { pre-molar }\end{array}$} & Baseline & $2.27 \pm 0.62$ & $1.79 \pm 0.53$ & $2.37 \pm 0.28$ & 6.37 & 0.003 \\
\hline & At 6 months & $2.53 \pm 0.61$ & $1.81 \pm 0.53$ & $2.34 \pm 0.28$ & 10.36 & $<0.001^{* * *}$ \\
\hline \multirow{2}{*}{$\begin{array}{c}\text { Mesial surface of right } \\
\text { mandibular central incisor }\end{array}$} & Baseline & $2.87 \pm 0.96$ & $2.04 \pm 0.71$ & $2.80 \pm 0.29$ & 12.58 & $<0.001^{* *}$ \\
\hline & At 6 months & $3.23 \pm 0.95$ & $2.07 \pm 0.71$ & $2.82 \pm 0.29$ & 9.04 & $<0.001^{* * *}$ \\
\hline
\end{tabular}


Table 2: Inter-comparison of mean PI between groups at baseline and at follow up

\begin{tabular}{|l|c|c|c|c|}
\hline Groups & \multicolumn{2}{|c|}{ Baseline } & \multicolumn{2}{c|}{ Follow up at 6 months } \\
\hline & Mean difference & $\boldsymbol{p}$ value & Mean difference & $\boldsymbol{p}$ value \\
\hline Group 1 VS Group 2 & 0.085 & 0.49 & 0.405 & $<0.001^{* *}$ \\
\hline Group 2 VS Group 3 & 0.083 & 0.57 & 0.015 & 0.97 \\
\hline Group 1 VS Group 3 & 0.001 & 1.00 & 0.390 & $<0.001^{* *}$ \\
\hline
\end{tabular}

Table 3: Inter-comparison of mean GI between groups at baseline and at follow up

\begin{tabular}{|l|c|c|c|c|}
\hline Groups & \multicolumn{2}{|c|}{ Baseline } & \multicolumn{2}{c|}{ Follow up at 6 months } \\
\hline & Mean difference & $\boldsymbol{p}$ value & Mean difference & $\boldsymbol{p}$ value \\
\hline Group 1 VS Group 2 & 0.01 & 0.98 & 0.02 & 0.91 \\
\hline Group 2 VS Group 3 & 0.00 & 0.98 & 0.07 & 0.34 \\
\hline Group 1 VS Group 3 & 0.00 & 1.00 & 0.09 & 0.91 \\
\hline
\end{tabular}

\section{Discussion}

Tobacco smoking, mostly in the form of cigarette smoking, is recognized as the most important environmental risk factor in periodontal diseases. This habit has proven to be an important risk factor involved in the initiation, maintenance, and aggravation of periodontal diseases in adults. (Offenbacher $\mathrm{S}$ et al.) ${ }^{1}$ These studies were mainly concerned with adult populations, generally heavy smokers with many years of cigarette consumption in whom other risk factors, such as age or systemic diseases, may co exist. The main findings of this prospective study were that smoking was associated with poorer periodontal condition as assessed clinically, and it had a negative effect on alveolar bone as assessed radiographically in a group of well-motivated young adults without periodontitis. Bergstrom et al. ${ }^{8}$ investigated the long-term( 10 years $)$ influence of smoking on periodontal bone height and demonstrated a reduction in bone height that was 2.7 times greater in adult smokers.

The effect of nicotine was studied in an animal model of periodontal disease by Fiore MC et al. ${ }^{9}$ The components of tobacco and nicotine metabolites may act directly as local irritants on the gingiva and alveolar bone or systemically (Offenbacher et al. $)^{1}$ Nicotine can suppress the proliferation of cultured osteoblasts while stimulating osteoblasts alkaline phosphatises activity. Slots $\mathbf{J}^{10}$ reported that nicotine increased the secretion of interleukin-6 (IL-6) and tumor necrosis factor alpha (TNF- $\alpha$ ) in osteoblasts. Nicotine also seems to stimulate bone matrix turnover by increasing the production of tissue-type plasminogen activator and matrix metalloproteinases (MMPs), there by tipping the balance between bone matrix formation and resorption toward the latter process, as reported by Katonoet al. ${ }^{11}$ Nicotine and lipopolysaccharide stimulated the formation of osteoclastlike cells via an increase in macrophage colony-stimulating factor and prostaglandin $\mathrm{E}_{2}\left(\mathrm{PGE}_{2}\right)$ production. Receptor activator of nuclear factor-kappa $\mathrm{B}$ ligand (RANKL) and osteoprotegerin (OPG) are members of the tumor necrosis factor super family. RANKL promotes osteoclastic differentiation and activates bone resorption. In contrast, OPG inhibits osteoclast genesis and suppresses bone resorption by inhibition of RANKL. ${ }^{12}$
Smoking cessation intervention is an important category in the dental practice. The dentist can assist the patient by offering medication and providing or referring for counselling or additional treatment, and arrange for followup contacts to prevent relapse (Tanaka et al.) ${ }^{13}$ Therefore, the provision of information about effective smoking cessation aids is an essential component of intervention for patients who are willing to quit.

The present study was designed to evaluate the effect of smoking and smoking cessation on alveolar bone in young adults and the Motivational Interviewing (MI) for the cessation of smoking. A total number of sixty patients having mean age of 23.4 years were participated in this study. They were categorized into three groups as described earlier. All patients from Group 1 and Group 2 successfully completed the study without any drop out, while six patients were dropped out from Group 3 at six months re-evaluation, as they refused to quit smoking.

The accumulation of dental plaque was higher for smokers than the other two groups. Inter-comparison of PI between groups at baseline and follow up showed that comparison between Group 1 and Group 2 showed statistically insignificant difference at baseline $(p=0.49)$, however at follow up it was found to be a statistically significant difference $(p<0.001)$. Comparison of PI at baseline and at follow up among all groups showed statistically insignificant at baseline, whereas it was found to be statistically highly significant at follow up. Similar results were reported by Al-Wahadni and Linden ${ }^{14}$ in a case control study of young Jordanians smokers.

Clinical gingival inflammation was almost the same in all three groups at baseline. After six months of follow up, a reduction in clinical gingival inflammation was observed in all groups, but Group 3 showed statistically highly significant result $(p<0.001)$. Studies have found greater (Gunsolley J C et al. $)^{15}$ and equal (Jin Q et al.) ${ }^{16}$ levels of gingival inflammation in smokers compared to nonsmokers. Al-Wahadni and Linden ${ }^{14}$ also found increased signs of gingival inflammation in young smokers.

The 6- to 12-month re-entry procedure has been the gold standard for the evaluation of changes in alveolar bone in most clinical regenerative studies during the last decades (Toback GA et al.). ${ }^{17}$ Despite the limitations of radiographic 
analysis, (Bragger U, Hausmann E) ${ }^{18,19}$ it remains the only non-invasive method for bone evaluation in young subjects with no periodontal destruction. Sopro ${ }^{\circledR}$ software was used for better identification of CEJ and measurements of alveolar bone loss along with RVG.

Alveolar bone height was measured in proximal sites of standardized, mandibular posteriors and mandibular anterior digital radiographs with a RVG. Group 1 had a decreased bone height at all stages of the study, and bone loss was noted in these subjects after six months of follow-up which is attributed to effect of smoking on alveolar bone, while Group $2 \&$ Group 3 had a constant alveolar bone height or slight decreased bone height at follow up except in group two right side distal to $1^{\text {st }}$ molar and mesial to $2^{\text {nd }}$ pre-molar which showed statistically insignificant result. The mean crestal bone loss of all seven surfaces was measured at baseline and at follow up. Statistically significant difference among Group 1, Group 2 \& Group 3 was found at right side mesial surface of $1^{\text {st }}$ molar $(p=0.001) \&$ left side mesial surface of $1^{\text {st }}$ molar $(p=0.002)$ at follow up. Statistically highly significant difference among Group 1, Group 2 \& Group $3(p<0.001)$ was found at right side distal surface of $1^{\text {st }}$ molar, right side mesial surface of $2^{\text {nd }}$ pre-molar, left side distal surface of $1^{\text {st }}$ molar, left side mesial surface of $2^{\text {nd }}$ premolar \& mesial surface of right mandibular central incisor at follow up. The statistical analysis disclosed that the duration of smoking had a negative effect on the alveolar bone height measured. These findings are in agreement with the literature (Haber $\mathbf{J}$ et al.), ${ }^{2}$ data from cross-sectional studies published by Feldman et al..$^{20}$ Bergstrom et al. ${ }^{8}$ and data from longitudinal studies published by Machtei et al. ${ }^{21}$ and Bergstrom et al. ${ }^{22}$ All of these studies were conducted in an older population (mean age $>39$ ) with a longer duration of smoking (mean $>10$ years). The current population under study was different because subjects were younger, and total smoking frequency was lower. Our results confirm previous reports about young smokers in cross sectional (Gunsolley JC et al. $)^{15}$ longitudinal clinical studies by Muller HP et al. ${ }^{23}$ and Levin $\mathrm{L}$ et al. ${ }^{24}$

The 5A approach provides a structure for identifying all smokers and offering support to help them quit (Mihir Shah et al. 2006). ${ }^{25}$ This model consists of five components for effective smoking cessation intervention: Ask about tobacco use; Advise about quitting; Assess willingness to make a quit attempt; Assist in the quit attempt; and Arrange followup.

In present study, MI was used for smoking cessation and at the end of six months; six patients were dropped out because they refused to quit the smoking. Very few studies demonstrate a dose-response relationship between risk reduction of periodontal disease and smoking cessation. Findings of the NHANES III (National Health and Nutrition Examination Survey) revealed that the odds of periodontitis for former smokers who quit $\geq 11$ years previously were indistinguishable from the odds for non-smokers (Tomar \& Asma). ${ }^{26}$

Smoking cessation was confirmed by the special screening kit (for cotinine) named Nicoscreen ${ }^{\circledR}$. It is a prompt screening tool used to detect cotinine in body fluids up to three days after the body has absorbed the substance. Although the subjects who participated in this study were all dental students, they correspond to a homogenous subset with regard to oral hygiene knowledge, periodontal health status, and social class. Smoking may impact alveolar bone negatively even in a well-motivated young population. The patterns of change on bone parameters evaluated over six months of follow-up might indicate that smoking is already damaging the alveolar bone in these subjects, who were clinically diagnosed to be free from destructive periodontal disease. Hashim $\mathrm{R}$ et $\mathrm{al}^{27}$ reported that smoking in adolescence was a strong predictor of loss of periodontal attachment occurring by the mid-twenties. Despite public information campaigns, a substantial proportion of the population continues to smoke.

\section{Conclusion}

Periodontal health is compromised by chronic smoking by an increase alveolar bone loss, whereas in non-smokers the periodontal health condition remained unaltered. The association between smoking and clinical morbidity tend to be dose-dependent. Importantly, the conditions in former smokers remained stable, very similar to those of nonsmokers. To conclude with, this study reveals that in smokers, more plaque score and reduced gingival inflammation is observed than non-smokers and smoking cessation group. It has negative impact on alveolar bone than non- smokers. Smoking cessation group and nonsmokers has better clinical parameter than smokers in terms of bone height. Smoking cessation is effective in preventing not only oral diseases but also the progression of periodontal tissue breakdown. Intervention may be integrated in existing procedures of dental treatment because improvement of outcome of the treatment is expected by smoking cessation. Nicoscreen ${ }^{\circledR}$ can be used as a prompt screening tool for smoking cessation.

\section{Source of funding}

None.

\section{Conflict of interest}

None.

\section{References}

1. Offenbacher S. Periodontal diseases: Pathogenesis. Ann Periodontol 1996;1:821-78.

2. Haber J, Wattles J, Crowley M, Mandell R, Joshipura K, Kent RL. Evidence for cigarette smoking as a major risk factor for periodontitis. J Periodontol 1993;64: 16-23.

3. Quinn SM, Zhang JB, Gunsolley JC, Schenkei HA, Tew JG. The influence of smoking and race on adult periodontitis and serum IgG $_{2}$ levels. J Periodontol 1998;69:171-7.

4. Albert DA, Anluwalia KP, Ward A, Sadowsky D. The use of 'academic detailing' to promote tobacco-use cessation counselling in dental offices. J Am Dent Assoc 2004;135:17006.

5. Walsh MM, Ellison JA. Treatment of tobacco use and dependence: the role of the dental professional. J Dent Educ 2005;69:521-37. 
6. Prochaska JO, Clemente CC, Norcross JC. In search of how people change applications to addictive behaviours. Am Psychol 1992;47:1102-14.

7. Kinane DF, Radvar M. The effect of smoking on mechanical and antimicrobial periodontal therapy. $J$ Periodontol 1997;68:467-72.

8. Bergstrom $\mathrm{J}$ and Eliason S. Cigarette smoking and alveolar bone height in subjects with a high standard of oral hygiene. $J$ Clin Periodontol 1987:14:466-9.

9. Fiore MC, Bailey WC, Cohen SJ, Dorfman SF, Goldstein MG (2008). Chapter 3: Clinical interventions for tobacco use and dependence, In: Treating tobacco use and dependence: clinical practice guideline. 2008 update, USDHHS, PHS, 37-62, Rockville, MD, USA.

10. Slots J. Position Paper: Systemic antibiotics in periodontics. American Academy of Periodontology. J Periodontol 2004;75:1553-65

11. Katono T, Kawato T, Tanabe N. Nicotine treatment induces expression of matrix metalloproteinase in human osteoblastic cells. Acta Biochem Biophys Sin (Shanghai) 2006;38:874-82.

12. Grossi SG, Zambon J, Machtei EE. Effects of smoking and smoking cessation on healing after mechanical periodontal therapy. J Am Dent Assoc 1997;128:599-607.

13. Tanaka H, Tanabe N, Shoji M. Nicotine and lippolysaccharide stimulate the formation of osteoclast-like cells by increasing macrophage colony stimulating factor and prostaglandin $\mathrm{E}_{2}$ production by osteoblasts. Life Sci 2006;78:1733-40.

14. Al-Wahadni A, Linden G.: The effects of cigarette smoking on the periodontal condition of young Jordanian adults. J Clin Periodontol 2003;30:132-7.

15. Gunsolley JC, Quinn SM, Tew J, Gooss CM, Brooks CN and Schenkein HA. The effect of smoking on individuals with minimal periodontal destruction. J Periodontol 1998;69:16570.

16. Jin Q, Cirelli JA, Park CH. RANKL inhibition through osteoprotegerin blocks bone loss in experimental periodontitis. J Periodontol 2007;78:1300- 8.

17. Toback GA, Brunsvold MA, Nummikoski PV, Masters LB, Mellonig JT, Cochran DL. The accuracy of radiographic methods in assessing the outcome of periodontal regenerative therapy. J Periodontol 1999;70:1479-89.

18. Bragger U. Digital imaging in periodontal radiography: A review. J Clin Periodontol 1988;15:551-7.

19. Hausmann E. A contemporary perspective on techniques for the clinical assessment of alveolar bone. J Periodontol 1990;61:149-56

20. Feldman RS, Bravacos JS, Rose CL. Association between smoking different tobacco products and periodontal disease indexes. J Periodontol 1983;54:481-7.

21. Machtei EE, Hausmann E, Schmidt M. Radiographic and clinical response to periodontal therapy. J Periodontol 1998;69:590-5.

22. Bergstrom J, Eliasson S, Hans Peber H. Cigarette smoking and Periodontal bone loss. J Periodontol 1991;62:242-6.

23. Muller HP, Stadermam S, Heinecke A. Longitudinal association between plaque and gingival bleeding in smokers and non-smokers. J Clin Periodontol 2002;29:287-94.

24. Levin L, Baev V, Lev R, Stabholz A, Ashkenazi M. Aggressive periodontitis among young Israeli armypersonnel. $J$ Periodontol 2006;77:1392-6.

25. Shah M, Cecily S, Arora M. Help your patients remain tobacco-free. May 2006.

26. Tomar SL, Asma S. Smoking-attributable periodontitis in the United States: findings from NHANES III. National Health and Nutrition Examination Survey. J Periodontol 2000:71:5:743-51.
27. Hashim R, Thomson WM, Pack AR. Smoking in adolescence as a predictor of early loss of periodontal attachment. Community Dent Oral Epidemiol 2001;29:130-5.

How to cite the article: Parikh HA, Shah MN, Shah SB, Shah PS, Patel RR. Evaluating the effects of smoking and smoking cessation on alveolar bone in young adults: An interventional prospective study. J Dent Specialities 2019;7(2):72-7. 